\title{
Prediction and Prevention of Postpolypectomy Bleeding: Necessity of a Different Approach for Patients Using Antithrombotic Agents
}

\author{
Duk Hwan Kim \\ Digestive Disease Center, CHA Bundang Medical Center, CHA University, Seongnam, Korea
}

See "Colonic Postpolypectomy Bleeding Is Related to Polyp Size and Heparin Use" by Flavia Pigò, Helga Bertani, Mauro Manno, et al., on page 287-292.

Since the adenoma-carcinoma sequence regarding colorectal cancer was revealed, endoscopic resection of precancerous lesions such as adenoma is recognized as the most important method with respect to colorectal cancer prevention. Generally, endoscopic polypectomy is a safe, easy, and convenient procedure. However, procedures are not always safe to perform. Several complications such as postcoagulation syndrome, perforation, and bleeding can occur despite best care. Among complications related with endoscopic polypectomy, postpolypectomy bleeding is the most common and undesirable situation. Although the results of each study differ slightly, the incidence of postpolypectomy bleeding is generally known to be $0.3 \%-6.1 \%$. ${ }^{1}$ However, considering the referral bias, the actual frequency is thought to be lower.

No consensus has been reached about the strategy to avoid postpolypectomy bleeding. First, prophylactic endoscopic management can be applied. Epinephrine containing saline injection, electric coagulation at the resection ulceration, prophylactic hemoclipping on the resection ulceration, and endoloop application are relatively popular strategies for postpolypectomy prevention. However, the effectiveness of

Received: April 10, 2017 Revised: April 14, 2017

Accepted: April 21, 2017

Correspondence: Duk Hwan Kim

Digestive Disease Center, CHA Bundang Medical Center, CHA University, 59 Yatap-ro, Bundang-gu, Seongnam 13496, Korea

Tel: +82-31-780-1925, Fax: +82-31-780-1876, E-mail: teires.d.kim@gmail.com

(cc) This is an Open Access article distributed under the terms of the Creative Commons Attribution Non-Commercial License (http://creativecommons.org/ licenses/by-nc/3.0) which permits unrestricted non-commercial use, distribution, and reproduction in any medium, provided the original work is properly cited. these preventive methods has not been established yet. A retrospective study that included 1,745 patients who underwent colonoscopic polypectomy showed no benefits of endoscopic preventive methods regarding postpolypectomy bleeding. ${ }^{2}$ Likewise, a meta-analysis study reported that prophylactic hemoclipping was not significant with respect to the prevention of postpolypectomy bleeding. ${ }^{3}$ Another study showed that argon plasma coagulation after polypectomy did not decrease delayed bleeding. ${ }^{4}$ On the contrary, a prospective randomized study that included 120 patients with 151 polyps reported that prophylactic submucosal injection before polypectomy decreased immediate postpolypectomy bleeding. ${ }^{5}$ Another research group that retrospectively analyzed 524 polyps in 463 patients showed that prophylactic hemoclipping reduced delayed postpolypectomy bleeding. ${ }^{6}$ Moreover, clinical importance is focused on delayed bleeding rather than on immediate bleeding. The most recent meta-analysis study showed that both mechanical and injection endoscopic preventive methods did not decrease delayed bleeding, whereas a prophylactic technique reduced immediate postpolypectomy bleeding. ${ }^{7}$

Various risk factors such as large size of the polyp, location on the right colon, use of antiplatelet or anticoagulation agents, and underlying comorbidities have been identified regarding postpolypectomy bleeding. Moreover, as the prevalence of cardiovascular/neurovascular disease is increasing with the aging of the population, antithrombotic agents are highly used recently. In addition, the numbers of newly developed antithrombotic agents and dual antiplatelet therapies are increasing. Current guidelines regarding invasive endoscopic 
procedure in patients receiving anticoagulation agents recommend using heparin as a bridging therapy in patients at high risk of thrombosis. However, the risk of postpolypectomy bleeding does not seem to decrease even if administration of the anticoagulation agent is interrupted. ${ }^{8}$ When postpolypectomy bleeding associated with heparin bridging therapy occurred, clinical presentation showed high incidence, late onset, and recurrent events that resulted in long-term hospitalization. ${ }^{9}$ In this issue of Clinical Endoscopy, Pigò et al. retrospectively analyzed postpolypectomy bleeding, focusing on antithrombotic therapy. ${ }^{10}$ Between 2007 and 2014, 118 patients with postpolypectomy bleeding were compared with 539 non-bleeding patients. Polyps larger than $10 \mathrm{~mm}$, heparin use, comorbidity, and presence of 2 or more risk factors were identified as risk factors of postpolypectomy bleeding in the multivariate analysis. These results suggest that the use of heparin as a bridge therapy alone cannot eliminate bleeding associated with polypectomy. A recent similar case-control study also demonstrated that an increased postpolypectomy bleeding incidence was observed regardless of antithrombotic agent discontinuation and use of heparin bridging therapy. ${ }^{11}$ Therefore, careful assessment of individual risk before polypectomy is warranted. Further study might attempt to more precisely classify the risk factors associated with various antithrombotic agents and to identify the preventive strategy related with each stratification.

\section{Conflicts of Interest}

The author has no financial conflicts of interest.

\section{REFERENCES}

1. Sorbi D, Norton I, Conio M, Balm R, Zinsmeister A, Gostout CJ. Postpolypectomy lower GI bleeding: descriptive analysis. Gastrointest Endosc 2000;51:690-696.

2. Kwon MJ, Kim YS, Bae SI, et al. Risk factors for delayed post-polypectomy bleeding. Intest Res 2015;13:160-165.

3. Boumitri C, Mir FA, Ashraf I, et al. Prophylactic clipping and post-polypectomy bleeding: a meta-analysis and systematic review. Ann Gastroenterol 2016;29:502-508.

4. Lee CK, Lee SH, Park JY, et al. Prophylactic argon plasma coagulation ablation does not decrease delayed postpolypectomy bleeding. Gastrointest Endosc 2009;70:353-361.

5. Hsieh YH, Lin HJ, Tseng GY, et al. Is submucosal epinephrine injection necessary before polypectomy? A prospective, comparative study. Hepatogastroenterology 2001;48:1379-1382.

6. Liaquat H, Rohn E, Rex DK. Prophylactic clip closure reduced the risk of delayed postpolypectomy hemorrhage: experience in 277 clipped large sessile or flat colorectal lesions and 247 control lesions. Gastrointest Endosc 2013;77:401-407.

7. Park CH, Jung YS, Nam E, Eun CS, Park DI, Han DS. Comparison of efficacy of prophylactic endoscopic therapies for postpolypectomy bleeding in the colorectum: a systematic review and network meta-analysis. Am J Gastroenterol 2016;111:1230-1243.

8. Witt DM, Delate T, McCool KH, et al. Incidence and predictors of bleeding or thrombosis after polypectomy in patients receiving and not receiving anticoagulation therapy. J Thromb Haemost 2009;7:1982-1989.

9. Inoue T, Nishida T, Maekawa A, et al. Clinical features of post-polypectomy bleeding associated with heparin bridge therapy. Dig Endosc 2014;26:243-249.

10. Pigò F, Bertani H, Manno M, et al. Colonic postpolypectomy bleeding is related to polyp size and heparin use. Clin Endosc 2017;50:287-292.

11. Kubo T, Yamashita K, Onodera K, et al. Heparin bridge therapy and post-polypectomy bleeding. World J Gastroenterol 2016;22:10009-10014. 